\title{
Intraclonal mating occurs during tsetse transmission of Trypanosoma brucei
}

\author{
Lori Peacock ${ }^{1,2}$, Vanessa Ferris ${ }^{1,2}$, Mick Bailey ${ }^{2}$ and Wendy Gibson*1
}

Address: ${ }^{1}$ School of Biological Sciences University of Bristol, Bristol BS8 1UG, UK and ${ }^{2}$ Department of Clinical Veterinary Science, University of Bristol, Langford, Bristol BS40 7DU, UK

Email: Lori Peacock - lori.peacock@bris.ac.uk; Vanessa Ferris -v.r.ferris@bris.ac.uk; Mick Bailey - mick.bailey@bris.ac.uk; Wendy Gibson* - w.gibson@bris.ac.uk

* Corresponding author

Published: 21 September 2009

Parasites \& Vectors 2009, 2:43 doi:10.1186/1756-3305-2-43
Received: 30 July 2009

Accepted: 21 September 2009

This article is available from: http://www.parasitesandvectors.com/content/2/1/43

(C) 2009 Peacock et al; licensee BioMed Central Ltd.

This is an Open Access article distributed under the terms of the Creative Commons Attribution License (http://creativecommons.org/licenses/by/2.0), which permits unrestricted use, distribution, and reproduction in any medium, provided the original work is properly cited.

\begin{abstract}
Background: Mating in Trypanosoma brucei is a non-obligatory event, triggered by the cooccurrence of different strains in the salivary glands of the vector. Recombinants that result from intra- rather than interclonal mating have been detected, but only in crosses of two different trypanosome strains. This has led to the hypothesis that when trypanosomes recognize a different strain, they release a diffusible factor or pheromone that triggers mating in any cell in the vicinity whether it is of the same or a different strain. This idea assumes that the trypanosome can recognize self and non-self, although there is as yet no evidence for the existence of mating types in T. brucei.
\end{abstract}

Results: We investigated intraclonal mating in $T$. b. brucei by crossing red and green fluorescent lines of a single strain, so that recombinant progeny can be detected in the fly by yellow fluorescence. For strain 1738, seven flies had both red and green trypanosomes in the salivary glands and, in three, yellow trypanosomes were also observed, although they could not be recovered for subsequent analysis. Nonetheless, both red and non-fluorescent clones from these flies had recombinant genotypes as judged by microsatellite and karyotype analyses, and some also had raised DNA contents, suggesting recombination or genome duplication. Strain JI0 produced similar results indicative of intraclonal mating. In contrast, trypanosome clones recovered from other flies showed that genotypes can be transmitted with fidelity. When a yellow hybrid clone expressing both red and green fluorescent protein genes was transmitted, the salivary glands contained a mixture of fluorescent-coloured trypanosomes, but only yellow and red clones were recovered. While loss of the GFP gene in the red clones could have resulted from gene conversion, some of these clones showed loss of heterozygosity and raised DNA contents as in the other single strain transmissions. Our observations suggest that many recombinants are non-viable after intraclonal mating.

Conclusion: We have demonstrated intraclonal mating during fly transmission of $T$. b. brucei, contrary to previous findings that recombination occurs only when another strain is present. It is thus no longer possible to assume that $T$. $b$. brucei remains genetically unaltered after fly transmission. 


\section{Background}

Genetic exchange and the production of hybrid trypanosomes can occur when two different strains of Trypanosoma brucei are co-transmitted through the tsetse fly vector $[1,2]$. In crosses to date, all subspecies of $T$. brucei have proved compatible, except $T$. b. gambiense group 1, excluded by its poor transmissibility in the commonlyused laboratory tsetse fly, Glossina morsitans morsitans [3]. Thus there would appear to be no subspecific barriers to mating, but nevertheless some kind of mating type restriction is thought to exist, because intraclonal mating occurs rarely and has been detected only in the presence of mating trypanosomes of different strains $[4,5]$. The hypothesis put forward is that some kind of diffusible factor or pheromone is produced by trypanosomes on recognition of non-self, which then triggers all trypanosomes in the vicinity to mate. Clearly, for this to work, trypanosomes must be able to recognize self and non-self, but mating types have not yet been described in T. brucei. A simple two-sex mating system was ruled out by the three-way cross carried out by Turner and colleagues [6], suggesting that the mating system of this diploid organism probably involves multiple mating types.

Previous studies on intraclonal mating have relied on genotyping individual clones after fly transmission, but the laborious and time-consuming nature of this work has limited the number of individual clones analysed, perhaps contributing to the failure to detect recombinants. For example, Tait et al [5] examined 45 metacyclic clones of seven T. brucei sspp. strains without finding recombinants. The use of different drug-selectable markers to distinguish two lines of the same strain also failed to reveal recombinants [4]; mixed infections were evident in two of 13 flies with infected salivary glands, but no double-drug resistant progeny were recovered.

Here we have revisited this problem using an approach that relies on the production of yellow fluorescent hybrids to indicate mating between different parental strains distinguished by red or green fluorescent proteins $[7,8]$. Using this robust experimental system for investigating genetic exchange in T. brucei, we previously demonstrated that mating took place only after trypanosomes had reached the salivary glands of the fly, and did not occur among trypanosomes in the midgut. As well as enabling hybrids to be detected by yellow fluorescence, the system makes it easy to identify which flies carry mixed populations since the two parental clones can be distinguished by red or green fluorescence. We have adapted the system to detect the occurrence of intraclonal mating by creating red and green fluorescent lines derived from a single trypanosome strain. The occurrence of yellow fluorescent trypanosomes when the red and green lines are co-transmitted through experimental tsetse flies should indicate intraclonal mating. Vice versa, transmission through tsetse flies of a yellow fluorescent trypanosome clone, which carries genes for both red and green fluorescence, would be expected to produce red and green fluorescent trypanosomes if the reporter genes segregated. Here we describe the results of both these kinds of experimental transmission.

\section{Methods \\ Tsetse flies}

Experimental tsetse flies were from the Bristol laboratory colony of Glossina morsitans morsitans originally from Zimbabwe. Flies were kept at $25^{\circ} \mathrm{C}$ and $70 \%$ relative humidity, and fed on sterile defibrinated horse blood via a silicone membrane; bloodmeals for infected flies were supplemented with $2.5 \% \mathrm{w} / \mathrm{v}$ bovine serum albumen (Sigma A4503) [9] and $1 \mathrm{mM}$ dATP [10]. Male flies were used for experiments, being given the infective bloodmeal for their first feed 24-48 hours post-eclosion. The infective bloodmeal contained approximately equal numbers of bloodstream form (BSF) trypanosomes of each strain (approximately $8 \times 10^{6}$ trypanosomes $\mathrm{ml}^{-1}$ ) in sterile horse blood, or procyclics in washed red blood cells (approximately $10^{7}$ trypanosomes per ml of packed cells), supplemented with $60 \mathrm{mM} \mathrm{N}$-acetylglucosamine [11] or $10 \mathrm{mM}$ L-glutathione [12] to increase infection rates.

\section{Trypanosomes}

The trypanosome clones used were 1738 (T. b. brucei MOVS/KE/70/1738 [13] and J10 (T. b. brucei MCRO/ZM/ 73/J10 CLONE 1 [14]) transfected with either a gene for green fluorescent protein (GFP) or monomeric red fluorescent protein $(m R F P)$ [15] as described previously [8]. A selectable marker gene, conferring resistance to the antibiotic hygromycin ( $\mathrm{Hyg}$ ) or phleomycin (Ble), was located downstream of the fluorescent protein gene in each plasmid construct. Constructs were targeted to the non-transcribed spacer of the ribosomal RNA locus. The clones used for mating were designated as follows according to fluorescence; the antibiotic resistance gene is given in brackets: 1738 GFP (Hyg), 1738 RFP (Ble), J10 GFP (Ble), J10 RFP $(\mathrm{Hyg})$. The intraclonal crosses are referred to as 1738 RGFP and J10 RGFP. A yellow fluorescent hybrid clone, SG3 clone 7, was also transmitted through tsetse. This hybrid clone originated from a cross of J10 RFP ( Hyg) and 1738 GFP $(\mathrm{Hyg})$ and had a DNA content similar to that of the parental trypanosomes, 2N [8]. Procyclic forms of SG3 clone 7 were transmitted through tsetse to obtain BSF, which were subsequently retransmitted; procyclic clones were derived from both BSF populations after transformation in vitro.

\section{Dissection}

Whole tsetse alimentary tracts, from the proventriculus to the hindgut, were dissected at various timepoints after 
feeding (21-54 days) in a drop of phosphate buffered saline (PBS) and scored for the presence of trypanosomes. A subset of these flies was used to quantify the proportion of mixed infections in the midgut by fluorescence microscopy; for the 1738 RGFP cross, trypanosomes were counted in midguts from a sample of flies dissected 21 to 32 days post infected feed, as described by [11]. Whole salivary glands from flies were dissected into a drop of PBS and viewed as wet mounts under bright field illumination to search for trypanosomes. Positive glands were then viewed by fluorescence microscopy using a DMRB microscope (Leica) equipped with a Colour Coolview camera (Photonic Science) and ImagePro Plus software (Media Cybernetics). Glands found to contain a mixed population of trypanosomes were digitally imaged before inoculation into mice. Trypanosomes from the first wave of parasitaemia were transformed to procyclics in vitro (see below).

\section{Trypanosome culture}

BSF trypanosomes from mice were transformed to procyclics in Cunningham's medium [16] supplemented with $10 \% \mathrm{v} / \mathrm{v}$ heat-inactivated foetal calf serum, $5 \mu \mathrm{g} / \mathrm{ml}$ hemin and $10 \mu \mathrm{g} / \mathrm{ml}$ gentamycin (complete medium $=\mathrm{CM}$ ) at $27^{\circ} \mathrm{C}$ and tested for drug resistance to hygromycin and/or phleomycin at $50 \mu \mathrm{g} \mathrm{ml}^{-1}$ and $1 \mu \mathrm{g} \mathrm{ml}^{-1}$ respectively in CM in a microtitre plate format. Plates were generally examined every 1 or 2 days for 2 weeks and individual wells passaged as necessary. Clones from transformed procyclics were obtained by limiting dilution in 96 well plates.

\section{Genotype analysis}

Genomic DNA samples were prepared from approximately $5 \times 10^{7}$ washed procyclics using a spin column DNA purification kit (Qiagen). Samples for pulsed field gel (PFG) electrophoresis were prepared by lysing and deproteinising trypanosomes in situ in agarose blocks [17]. PFG electrophoresis, blotting and hybridization were carried out essentially as described previously [8]. Chromosomes were separated using a Biorad CHEF-DR III with a 2 phase program (Block 1: switch time $1800 \mathrm{~s}$, voltage $2 \mathrm{~V} / \mathrm{cm}$, angle $106^{\circ}, 15$ hours; Block 2: switch time $300-900 \mathrm{~s}$, voltage $3 \mathrm{~V} / \mathrm{cm}$, angle $106^{\circ}, 50$ hours) using $0.5 \times$ TBE buffer and $0.9 \%$ agarose gels; gels were stained overnight by submersion in electrophoresis buffer containing ethidium bromide $(2 \mu \mathrm{g} / \mathrm{ml})$. Blots were hybridized with the following P32-labelled DNA fragments: GFP and $m R F P$ genes from the plasmid constructs used for transfection; $\beta$-tubulin from cDNA plasmid clone [18]; $18 \mathrm{~S}$ rRNA, trypanothione synthetase (TS; chromosome II), paraflagellar rod protein (PFR1, chromosome III) genes from T. brucei genomic DNA [19]. Microsatellite analysis was carried out using eight chromosome-specific primer sets - see Tables 3, 4, 5[20]. Primer sets for each locus were as follows: II-PLC 5' CAACGACGTTGGAA-
GAGTGTGAAC， 5' CCACTGACCTTTCATTTGATCGCTT TC; III-2 5' GGTGGAATGGAAGATCAGTT, 5' GTTGGAAT TGTTGTTGCTGT; IV-TB4/7 5' CCGTCACACGCCATGCACGATATG，5' CCGTTCAGTGTGCATGTTTCAC; VITB6/1 5' CATGATGCGGAACACATGACC， 5' CTCAATAGTGCAAGTAGTCATAC; VIII-TB8/9 5' CCAAATATGCGATTAGTTTCC，5' TGTTTATGTGGAAGGAAATGAA; IX-TB9/6 5' AAGTGTGAGGAGTTGTTGT, 5' CACCCCTTTCATCAACATCAT; X-TB10/17 5' CTCGCACGTTCGGATTTATGTCCG，5' GCGACTTGTGACTTGCCTTTCTTC; XI-53 5' CGTGTGTCTTGTATATCTTCT， 5' TGAATAA ACAAAACATGAAACGAC. Products were resolved by electrophoresis in $1 \times$ TAE buffer through 3-5\% Metaphor agarose (Cambrex) gels.

\section{Measurement of DNA contents}

DNA contents of individual clones were measured by flow cytometry of fixed procyclics stained with propidium iodide as described [8]. Measurements were standardized by reference to the DNA content of T. b. brucei strain 427, arbitrarily assigned a value of 1.00 ; procyclics of strain 427 were included in every batch of samples run.

\section{Results and Discussion}

\section{Analysis of experimental cross I738 RGFP}

For the cross 1738 RGFP, a total of 461 flies were coinfected with approximately equal numbers of BSF trypanosomes of 1738 RFP and 1738 GFP and dissected in batches 21-54 days later. The overall midgut infection rate was $43 \%(197 / 461)$; in a subset of these fly midguts examined by fluorescence microscopy, the majority contained a mixture of red and green fluorescent trypanosomes (78/ $88=89 \%)$, with $8 \%(7 / 88)$ having only green and $3 \%(3 /$ 88 ) only red fluorescent trypanosomes. Counts of trypanosomes in midguts dissected between 21 and 32 days after infection showed no significant difference in numbers of green $\left(1.54 \times 10^{5} \pm 2.75 \times 10^{4}\right)$ and red fluorescent trypanosomes $\left(1.61 \times 10^{5} \pm 1.90 \times 10^{4}\right)(t=0.247$, $\mathrm{df}=18, P=0.808, n=19$, Paired-samples $t$-test). There was thus no detectable difference in the ability of these two recombinant clones of strain 1738 to establish infection in the fly midgut.

There were a total of 22 salivary gland infections, giving a transmission index of $11 \%(22 / 197)$. In seven flies, the salivary glands contained a mixed infection of red and green fluorescent trypanosomes, while the remainder had trypanosomes of only a single colour (Table 1). Small numbers of yellow trypanosomes were seen in the salivary glands of three of the seven flies with a mixed salivary gland infection (Table 2; Fig 1). This contrasts with the abundance of yellow fluorescent trypanosomes typically observed in salivary glands containing both 1738 GFP and J10 RFP in the previous interclonal cross [8]. 
Table I: Salivary gland infections

\begin{tabular}{llll}
\hline Cross & \multicolumn{2}{l}{ Fluorescence of trypanosomes in paired salivary glands (no. of flies) } & Total \\
\cline { 2 - 4 } & Red only & Green only & Red + Green \\
\hline I738 RGFP & 2 & 13 & 7 \\
\hline J10 RGFP & 9 & 2 & 2 \\
\hline
\end{tabular}

Metacyclics from the seven flies with mixed salivary gland infections were transformed to BSF in rodents and then into procyclic populations in vitro to facilitate drug selection and cloning. No yellow fluorescent trypanosomes were observed in any of the procyclic populations, with a mixture of red and green fluorescent trypanosomes evident in only two populations (Fly 6 and 7; Table 2). Growth with antibiotics showed drug resistance consistent with the observed fluorescence of the procyclic populations, but no evidence of double-drug resistant trypanosomes (Table 2). Clones were derived from populations 1, 6 and 7, all of which originated from salivary glands with red, green and yellow fluorescent trypanosomes (Table 2). Of 20 clones analysed, four from Fly 6 and 7 lacked fluorescence, while the rest were either red or green (Table 3). These clones were genotyped by microsatellite analysis of eight chromosome-specific loci (Table
3). While all clones from Fly 1 and 6 matched the 1738 genotype, five clones from Fly 7 showed homozygosity at loci where 1738 was heterozygous, indicating that they are recombinant (Table 3; Fig 2A and 2B). In each case, homozygosity was demonstrated at 3-4 independent loci, making it unlikely that these results can be explained by gene conversion.

This interpretation was supported by analysis of molecular karyotypes by PFG electrophoresis. Inspection of the ethidium-stained gel revealed small differences among clones from Fly 7, while clones from Fly 1 and 6 were indistinguishable from 1738 GFP or 1738 RFP; Fig 3A shows a comparison of clones from Fly 6 and 7. The variability of Fly 7 clones was confirmed by hybridization of the PFG blot with a probe for 18S rDNA (Fig 3B), which is carried on several different chromosomes (rDNA arrays

Table 2: Composition of trypanosome population in individual flies

\begin{tabular}{|c|c|c|c|c|c|c|c|c|c|c|c|}
\hline \multirow[t]{3}{*}{ Cross } & \multirow[t]{3}{*}{ Fly no. } & \multicolumn{6}{|c|}{ Fluorescence of salivary gland population ${ }^{\mathrm{a}}$} & \multirow[t]{3}{*}{$\begin{array}{l}\text { Fluoroscence of } \\
\text { transformed procyclics }\end{array}$} & \multicolumn{3}{|c|}{$\begin{array}{l}\text { Drug resistance of } \\
\text { population }\end{array}$} \\
\hline & & \multicolumn{4}{|c|}{ Gland I } & \multicolumn{2}{|c|}{ Gland 2} & & \multirow[b]{2}{*}{$\mathbf{H}$} & \multirow[b]{2}{*}{$\mathbf{P}$} & \multirow[b]{2}{*}{$\mathbf{H}+\mathbf{P}$} \\
\hline & & Red & Green & Yellow & Red & Green & Yellow & & & & \\
\hline \multirow[t]{7}{*}{ I738 RGFP } & 1 & ++ & +++ & $+/-$ & ++ & +++ & $+/-$ & Red & - & + & - \\
\hline & 2 & + & +++ & - & + & +++ & - & Green & + & - & - \\
\hline & 3 & $+/-$ & +++ & - & $+/-$ & +++ & - & Green & + & - & - \\
\hline & 4 & $+/-$ & +++ & - & $+/-$ & +++ & - & Green & + & - & - \\
\hline & 5 & $+/-$ & +++ & - & $+/-$ & +++ & - & Green & + & - & - \\
\hline & 6 & ++ & +++ & + & +++ & ++ & + & Green, Red & + & + & - \\
\hline & 7 & $+/-$ & +++ & - & +++ & +++ & + & Green, Red & + & + & - \\
\hline \multirow[t]{2}{*}{ JI0 RGFP } & 1 & - & - & +++ & +++ & - & - & Red & + & - & - \\
\hline & 2 & + & +++ & - & +++ & +++ & - & Green, Red & + & + & - \\
\hline
\end{tabular}

a. Only flies with mixed infections in the salivary glands are listed. +++ many, ++ some, + few, +/- very few, - no trypanosomes.

b. $\mathrm{H}=$ hygromycin; $\mathrm{P}=$ phleomycin. + growth, - no growth. 

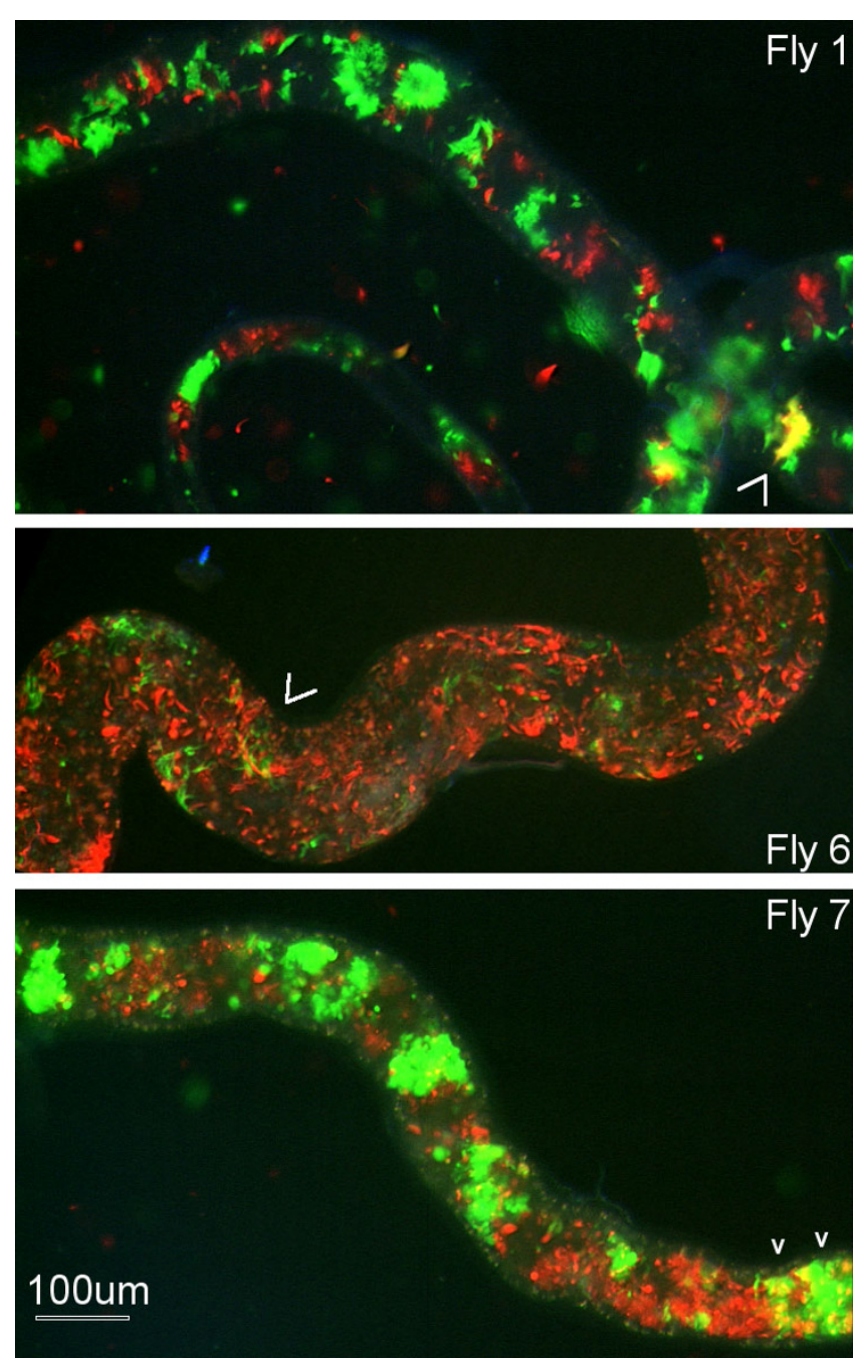

Figure I

Infected salivary glands 1738 RGFP cross. Dissected salivary glands from three flies from 1738 RGFP cross with mixed infection of red, green and yellow trypanosomes. Arrows point to an area of gland containing yellow trypanosomes.

on chromosomes II, III and VII [19]), and a series of chromosome-specific probes. For the PFR1 locus on chromosome III, where the two homologues of 1738 differ substantially in size ( 1.7 and $2.2 \mathrm{Mb}$ ), only a single band was evident in Fly 7 clones 1, 2 and 6 (Fig 3C). As both alleles $c$ and $d$ were detected in these clones for microsatellite locus III-2 (Table 3), two chromosome III homologues of the same size are present; moreover, as allele $c$ is normally located on the smaller of the two chromosome III homologues in strain 1738, but is now on a large homologue, chromosomal recombination has occurred. Further evidence of chromosomal recombination is the presence of two copies of the mRFP gene in Fly 7 clone 2, while only a single copy is present in 1738 RFP and other red fluorescent clones (Fig $3 \mathrm{D}$ and $3 \mathrm{E}$ ). The $m R F P$ gene
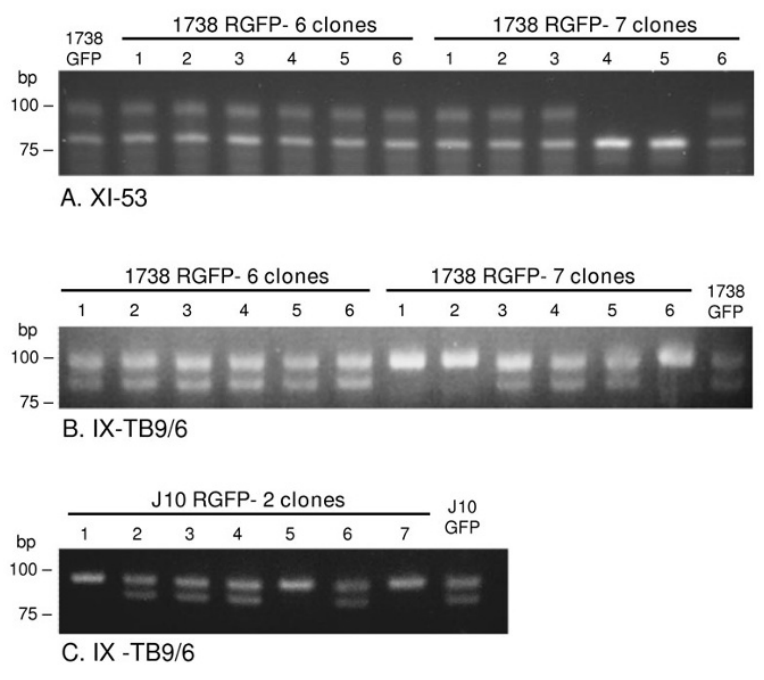

Figure 2

Homozygosity demonstrated by microsatellite analysis. PCR-amplified products for microsatellite loci indicated for the crosses 1738 RGFP (panels A and B) and JIO RGFP (panel C). Only the GFP parental clone is shown in each panel as the RFP clone was identical in each case. A. 1738 RGFP-7 clones 4 and 5 are homozygous, whereas all other clones and the parental clone, I738 GFP, are heterozygous. B. I738 RGFP-7 clones I, 2 and 6 are homozygous, whereas all other clones and the parental clone, 1738 GFP, are heterozygous. C. JIO RGFP-2 clones I, 5 and 7 are homozygous, whereas all other clones and the parental clone, JI0 GFP, are heterozygous.

was targeted to integrate into the non-transcribed spacer region of one of the rDNA arrays, and co-localization with a probe for the trypanothione synthetase (TS) gene showed the recombinant array to be on chromosome II (Fig 3F). While the chromosome II homologues of strain 1738 are approximately equal in size $(\sim 1.2 \mathrm{Mb})$, one homologue is much larger $(\sim 1.8 \mathrm{Mb})$ in Fly 7 clone 2 and both homologues carry the mRFP gene (Fig $3 \mathrm{E}$ and $3 \mathrm{~F}$ ).

Measurement of DNA contents by flow cytometry relative to strain 427 gave values of 0.91 for 1738 RFP and 1738 GFP, and $0.90-0.97$ for the clones analysed from Fly 1 and 6 (Table 3). Four clones from Fly 7 also fell within or just outside this range, but clones 1 and 3 had substantially higher values of 1.17 and 1.21 respectively (Table 3 ). While there was evidence from microsatellite and karyotype data that Fly 7 clone 1 was recombinant, clone 3 showed no difference to the profile of 1738 except lack of fluorescence (Table 3).

Taken together, the microsatellite and karyotype data identify five clones $(1,2,4,5,6)$ from Fly 7 as products of intraclonal mating of strain 1738. The increased DNA 
Table 3: Genotypes and DNA contents of clones from I 738 RGFP cross

\begin{tabular}{|c|c|c|c|c|c|c|c|c|c|c|}
\hline \multirow[t]{2}{*}{ Clone } & \multirow[t]{2}{*}{ Fluorescence } & \multirow[t]{2}{*}{ DNA content ${ }^{\mathrm{a}}$} & \multicolumn{8}{|c|}{ Microsatellite locus ${ }^{b}$} \\
\hline & & & II-PLC & III-2 & IV-TB4/7 & VI-TB6/I & VIII-TB8/9 & IX-ТВ9/6 & X-ТВ $10 / 17$ & XI-53 \\
\hline I738 RFP & Red & 0.91 & cd & cd & cd & bc & bc & cd & bc & bc \\
\hline 1738 GFP & Green & 0.91 & cd & $c d$ & cd & bc & bc & cd & bc & bc \\
\hline Fly I clones I-8 & Red & 0.90 & cd & cd & $\mathrm{cd}$ & bc & bc & cd & bc & bc \\
\hline Fly 6 clone I & None & 0.97 & cd & cd & cd & bc & bc & cd & bc & bc \\
\hline Fly 6 clone 2 & Green & 0.93 & cd & $\mathrm{cd}$ & $\mathrm{cd}$ & bc & bc & $\mathrm{cd}$ & bc & bc \\
\hline Fly 6 clone 3 & Green & 0.90 & cd & cd & $\mathrm{cd}$ & bc & bc & cd & bc & bc \\
\hline Fly 6 clone 4 & Green & 0.90 & $\mathrm{~cd}$ & $\mathrm{~cd}$ & $\mathrm{~cd}$ & bc & bc & cd & bc & bc \\
\hline Fly 6 clone 5 & Red & 0.91 & $\mathrm{~cd}$ & $\mathrm{~cd}$ & $\mathrm{~cd}$ & bc & bc & $\mathrm{cd}$ & bc & bc \\
\hline Fly 6 clone 6 & Green & 0.96 & cd & cd & $\mathrm{cd}$ & bc & bc & cd & $\mathrm{bc}$ & bc \\
\hline Fly 7 clone I & Red & 1.21 & $\mathrm{~cd}$ & cd & cc & cc & bc & dd & bb & bc \\
\hline Fly 7 clone 2 & Redc & 0.98 & $\mathrm{~cd}$ & $\mathrm{~cd}$ & cc & cc & bc & dd & bb & bc \\
\hline Fly 7 clone 3 & None & 1.17 & cd & cd & cd & bc & bc & cd & bc & bc \\
\hline Fly 7 clone 4 & None & 0.96 & $\mathrm{~cd}$ & cd & cc & bb & bc & $\mathrm{cd}$ & bc & bb \\
\hline Fly 7 clone 5 & None & 0.95 & $\mathrm{~cd}$ & $\mathrm{~cd}$ & cc & bb & bc & $\mathrm{cd}$ & bc & bb \\
\hline Fly 7 clone 6 & Red & 0.97 & cd & $\mathrm{cd}$ & cc & cc & bc & dd & bb & bc \\
\hline
\end{tabular}

a. DNA content relative to T. b. brucei strain 427, arbitrarily assigned a DNA content of I.00. For Fly I, value given is for clone I.

b. Primers for microsatellite loci on chromosomes II, III and XI given in [8], and on chromosomes IV, VI, VIII, IX and X from database of Dr Annette Macleod http://www.gla.ac.uk/centres/wcmp/research/macleod/trypanosomegeneticmappingdatabase/ text indicates differences from alleles of $T$. $b$. brucei strain 1738.

c. Two copies of mRFP gene

content of Fly 7 clone 3 also indicates that it is a recombinant. Clones 4 and 5 were identical on all criteria (Table $3)$, hence there are five individual genotypes from Fly 7 that result from intraclonal mating. This contrasts with the absolute fidelity of transmission of 1738 RFP and 1738 GFP as demonstrated by the recovery of unaltered clones from Fly 1 and 6.

\section{Analysis of experimental cross JI0 RGFP}

For the cross J10 RGFP, a total of 407 flies were co-infected with approximately equal numbers of BSF trypanosomes of J10 RFP and J10 GFP and dissected in batches 33-41 days later. The overall midgut infection rate was $64 \%$ $(259 / 407)$ and the majority of midguts in a subset examined by fluorescence microscopy contained a mixture of red and green fluorescent trypanosomes $(51 / 62,82 \%)$, with $16 \%(10 / 62)$ having only red and $2 \%(1 / 62)$ only green fluorescent trypanosomes. There were 13 salivary gland infections, giving a transmission index of 5\% (13/ 259). For 11 of these flies, trypanosomes of single colour fluorescence were detected in both salivary glands (Table 1) and these were not analysed further. Of the two remaining flies, Fly 1 had one salivary gland packed with yellow fluorescent trypanosomes; the colour of fluorescence was verified using narrow bandwidth red and green filters (Fig 4); the other gland contained only red trypanosomes. Although we assume that yellow fluorescent metacyclics were present in the salivary gland, disappointingly no yellow fluorescent trypanosomes were observed in the procyclic population derived from the glands after mouse passage, which comprised red fluorescent trypanosomes only, and no double drug resistant 


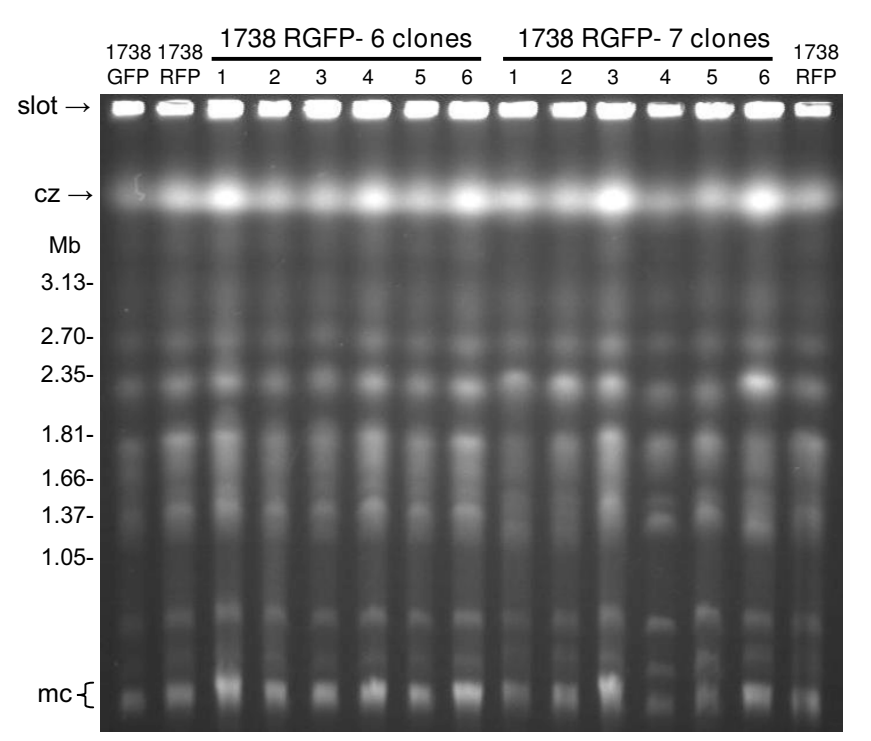

A.

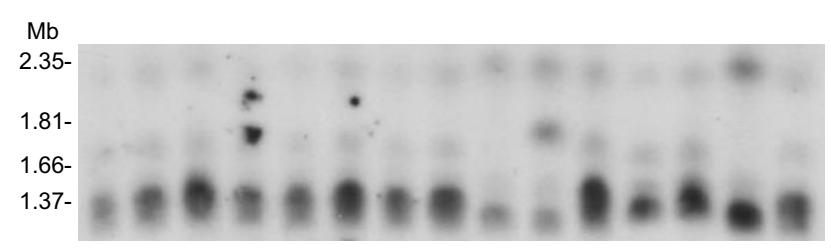

B. $18 S$ rDNA

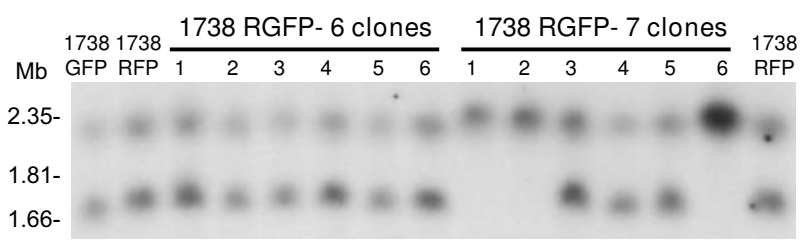

\section{PFRI chromosome III}

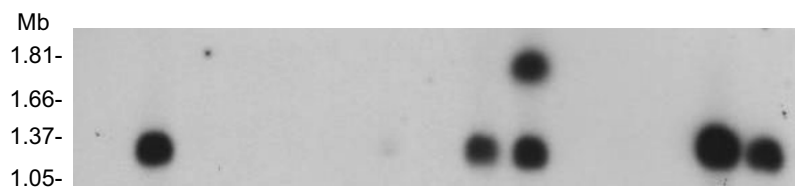

D. $m R F P$

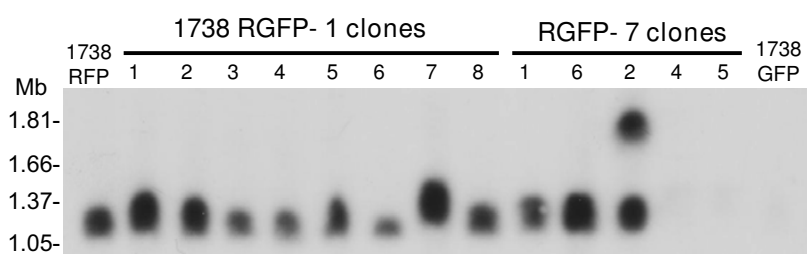

E. $m R F P$

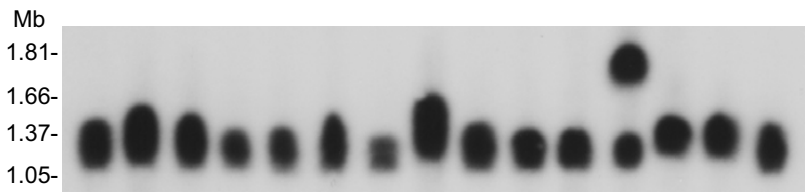

F. TS chromosome II

\section{Figure 3}

Molecular karyotypes of clones from 1738 RGFP cross. A. Ethidium bromide stained pulsed field gel. Size marker: chromosomal DNAs from Hansenula wingei. $\mathrm{Cz}=$ compression zone, a region of the gel where several large chromosomal bands are trapped. Mc $=$ minichromosomes of approx. $100 \mathrm{~kb}$ in size. B - D. Autoradiographs of Southern blot of same gel showing results following hybridization with the probes indicated. All blots were washed to $0.1 \times$ SSC at $65^{\circ} \mathrm{C}$. E, F. Southern blot of second gel (not shown) hybridised with probes indicated. Chromosome III homologues are identical in size in Fly 7 clones I, 2 and 6 , while clone 2 has two copies of the mRFP gene.

trypanosomes were recovered by antibiotic selection (Table 2); BSF were not checked for fluorescence, as the fluorescent reporter genes are driven by a procyclin promotor, which is down-regulated in BSF. Fly 2 had a mixed infection of red and green fluorescent trypanosomes in both glands, but no yellow fluorescent trypanosomes were noticed, nor were double drug resistant trypanosomes recovered after antibiotic selection (Table 2).

Clones were obtained from Fly 1 and 2 procyclic populations prior to drug selection; all eight clones from Fly 1 showed red fluorescence, while those from Fly 2 showed either red or green fluorescence (Table 4). Analysis of molecular karyotypes did not reveal any differences from J10 (not shown). However, several clones showed homozygosity for the microsatellite locus on chromosome IX whereas J10 was heterozygous (Fig 2C; Table 4). A difference between J10 GFP and J10 RFP was detected for the microsatellite locus on chromosome VIII, while all clones had $a b$ alleles like J10 GFP, regardless of colour of fluorescence (Table 4); we assume J10 RFP became homozygous during the selection and fly transmission involved in derivation of the lineage.

Measurement of DNA contents by flow cytometry relative to strain 427 gave values of 0.96 for J10 RFP and J10 GFP, but all clones from Fly 1 had greatly increased DNA contents, ranging from 1.48 to 1.76 (Table 4). Based on the haploid DNA content of J10 (0.48), these values indicate triploid cells. Fly 2 clone 1 also had a high DNA content, while the other clones measured had similar values to J10 (0.93 - 1.05; Table 4). The raised DNA contents of some clones from Fly 1 and 2, coupled with reduction to homozygosity at one microsatellite locus, suggest that these clones are products of intraclonal mating. This evidence is strengthened by the striking observation of widespread yellow fluorescence in one salivary gland of Fly 1 (Fig 4). This deserves comment, as such predominant yel- 

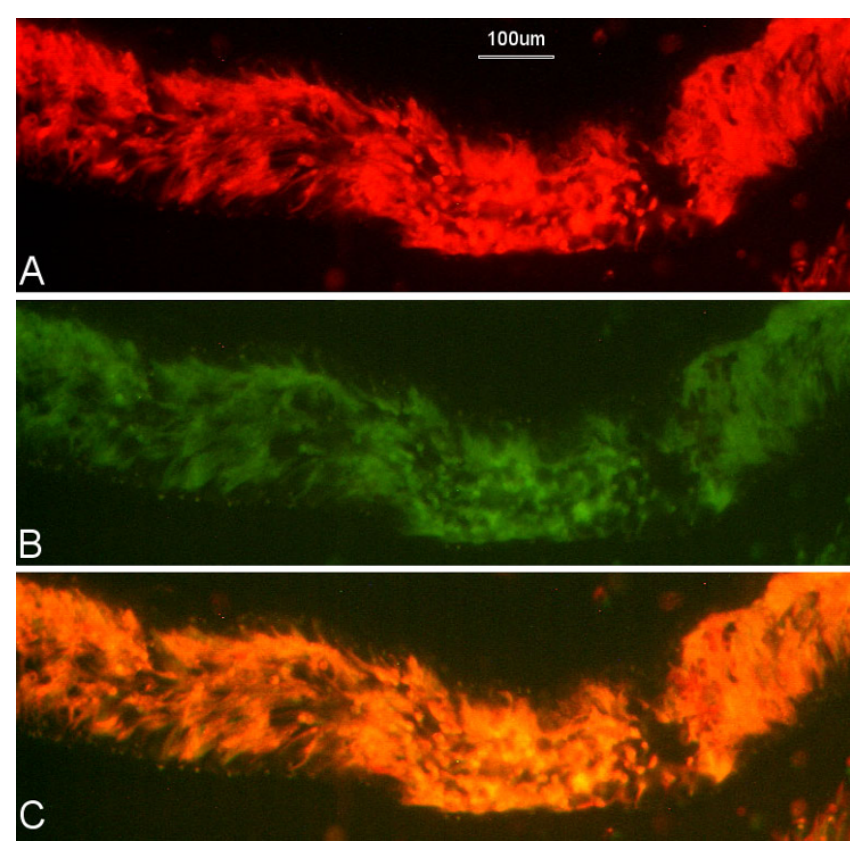

Figure 4

Infected salivary gland $\mathbf{J} 10$ RGFP cross. Portion of dissected salivary gland from JIO RGFP-I containing predominantly yellow fluorescent trypanosomes. Viewed through red filter (A) or green filter (B), and merge (C).

low fluorescence has never been observed even in salivary glands from red/green crosses. It is possible that trypanosome fusion took place on a large scale in this gland for some reason, but this implies that large numbers of red and green fluorescent trypanosomes were already present; on the other hand, if the gland was colonized by a small population of yellow fluorescent trypanosomes, this implies that there was an actively dividing population, which does not square with the subsequent failure to recover any viable yellow trypanosomes.

\section{Transmission of a yellow fluorescent clone}

SG3 clone 7 is a hybrid clone derived from the 1738 GFP $\times$ J10 RFP cross; it expresses both $m R F P$ and GFP genes and therefore appears yellow by fluorescence microscopy [8]. Fly transmission of this clone was carried out twice in succession, first using SG3 clone 7 procyclics in the infective bloodmeal. Three flies with infected salivary glands were obtained, but the trypanosomes were not of uniform yellow fluorescence as expected, instead showing a range of fluorescent colours including green, red and yellow (Fig 5); most surprisingly, one fly had one salivary gland of the pair containing only red fluorescent trypanosomes. The infected glands from these three flies were used to obtain BSF populations, which were transformed to procyclics in vitro; no green fluorescent trypanosomes were seen in this population and the red and yellow fluorescent clones obtained were analysed by microsatellite and karyotype analysis (Table 5, Transmission 1). No differences were detected in the genotypes of any of the clones except for the absence of the GFP gene in the red fluorescent clones (Fig 6B). The GFP gene was on the smaller homologue of chromosome VII in the yellow fluorescent clones; hybridization with a probe for $18 \mathrm{~S}$ rDNA showed both chromosome VII homologues present and unchanged in size in all clones (not shown). The most likely explanation of these observations is that the GFP gene was removed by gene conversion, because it sits in a tandem array of rDNA repeats. However, measurement of DNA contents of two red and two yellow clones showed a range of values: while yellow clones, 7A1 and 2, both gave values of about 0.9 and are probably diploid, the two red clones had either a very high $(1.58,7 \mathrm{D} 1)$ or low $(0.78,7 \mathrm{D} 4)$ value (Table 5$)$. Previously SG3 clone 7 was found to have a DNA content similar to that of its parents, 1738 GFP and J10 RFP, consistent with diploidy [8].

The two yellow clones (7A1 and 7A2) were re-transmitted through flies. Of 39 flies with infected salivary glands, all except one had salivary glands with trypanosomes of red, green or yellow fluorescence as on the first transmission of the parent clone, SG3 clone 7; the exception was one fly that had only red trypanosomes in both glands. Salivary glands from six flies, representing transmission of clone 7A1 (7A1-1 to 4) and 7A2 (7A2-1 and 2) were used to obtain bloodstream form populations, which were transformed to procyclics in vitro. As before, no green fluorescent trypanosomes were observed in the population and only yellow or red clones were obtained for microsatellite and karyotype analysis (Table 5, Transmission 2). These clones were again indistinguishable by molecular karyotype (not shown) and most were identical by microsatellite analysis, but 7A1-4 clone 1 had become homozygous at two previously heterozygous loci and it also showed a high DNA content (1.42, Table 5). A second clone from this fly (7A1-4 clone 2) also showed homozygosity at a single microsatellite locus, but was lost prior to measurement of DNA content.

One interpretation of these results is that, like its parents 1738 and J10, SG3 clone 7 also undergoes intraclonal mating, leading to the occurrence of pure red and green fluorescent trypanosomes as well as yellow ones in the salivary glands; it is an open question whether the red and green trypanosomes are haploid intermediates or diploid progeny. As most salivary glands had this appearance, SG3 clone 7 may have a higher frequency of intraclonal mating than either parental strain, but this may also reflect the fact that these events are easy to detect in a yellow clone that carries genes for both red and green fluorescence. If so, it is puzzling that these high levels of intraclonal mating did not feed through to recovery of 
Table 4: Genotypes and DNA contents of clones from JIO RGFP cross

\begin{tabular}{|c|c|c|c|c|c|c|c|c|c|c|}
\hline \multirow[t]{2}{*}{ Clone } & \multirow[t]{2}{*}{ Fluorescence } & \multirow[t]{2}{*}{ DNA content $\mathrm{t}^{\mathrm{a}}$} & \multicolumn{8}{|c|}{ Microsatellite locus ${ }^{b}$} \\
\hline & & & II-PLC & III-2 & IV-TB4/7 & VI-TB6/I & VIII-TB8/9 & IX-TB9/6 & X-TB $10 / 17$ & XI-53 \\
\hline JIO RFP & Red & 0.96 & $a b$ & $a b$ & $a b$ & aa & $\mathrm{aa}^{\mathrm{c}}$ & $a b$ & $a b$ & aa \\
\hline JI0 GFP & Green & 0.96 & $a b$ & $a b$ & $a b$ & aa & $a b$ & $a b$ & $a b$ & aa \\
\hline Fly I clone I & Red & 1.61 & $a b$ & $a b$ & $a b$ & aa & $\mathbf{a b}$ & bb & $a b$ & aa \\
\hline Fly I clone 2 & Red & 1.64 & $a b$ & $a b$ & $a b$ & aa & $\mathbf{a b}$ & bb & $a b$ & aa \\
\hline Fly I clone 3 & Red & 1.51 & $a b$ & $a b$ & $a b$ & aa & $\mathbf{a b}$ & bb & $a b$ & aa \\
\hline Fly I clone 4 & Red & 1.48 & $a b$ & $a b$ & $a b$ & aa & $\mathbf{a b}$ & bb & $a b$ & aa \\
\hline Fly I clone 5 & Red & 1.76 & $a b$ & $a b$ & $a b$ & aa & $\mathbf{a b}$ & bb & $a b$ & aa \\
\hline Fly I clone 6 & Red & 1.55 & $a b$ & $a b$ & $a b$ & aa & $\mathbf{a b}$ & bb & $a b$ & aa \\
\hline Fly I clone 7 & Red & 1.76 & $a b$ & $a b$ & $a b$ & aa & $a b$ & bb & $a b$ & aa \\
\hline Fly I clone 8 & Red & ND & $a b$ & $a b$ & $a b$ & aa & $a b$ & bb & $a b$ & aa \\
\hline Fly 2 clone I & Red & 1.77 & $a b$ & $a b$ & $a b$ & aa & $\mathbf{a b}$ & bb & $a b$ & aa \\
\hline Fly 2 clone 2 & Green & 1.05 & $a b$ & $a b$ & $a b$ & aa & $a b$ & $a b$ & $a b$ & aa \\
\hline Fly 2 clone 3 & Green & 0.99 & $a b$ & $a b$ & $a b$ & aa & $a b$ & $a b$ & $a b$ & aa \\
\hline Fly 2 clone 4 & Green & ND & $a b$ & $a b$ & $a b$ & aa & $a b$ & $a b$ & $a b$ & aa \\
\hline Fly 2 clone 5 & Red & 0.97 & $a b$ & $a b$ & $a b$ & aa & $a b$ & bb & $a b$ & aa \\
\hline Fly 2 clone 6 & Green & 0.93 & $a b$ & $a b$ & $a b$ & aa & $a b$ & $a b$ & $a b$ & aa \\
\hline Fly 2 clone 7 & Red & ND & $a b$ & $a b$ & $a b$ & aa & $\mathbf{a b}$ & bb & $a b$ & aa \\
\hline
\end{tabular}

a. DNA content relative to T. b. brucei strain 427, arbitrarily assigned a DNA content of I.00. ND, not done.

b. See Table 4 for information on microsatellite loci.

c. There is an allelic difference between JIO RFP and JI0 GFP, presumably generated during derivation of these separate lineages.

large numbers of recombinant progeny. Like the predominance of yellow fluorescent trypanosomes in one salivary gland for J10, the observation of salivary glands filled with red fluorescent trypanosomes in sequential transmissions of SG3 clone 7 is difficult to explain. Do these uniform populations arise by founder effects [21], or are they evidence of widespread recombination? In considering these alternatives, it is noteworthy that red and green fluorescent trypanosomes were also observed in small numbers in the midguts of infected flies.

\section{Conclusion}

Contrary to previous reports that intraclonal mating in Trypanosoma brucei is only associated with out-crossing
$[4,5]$, we show here that recombinant trypanosomes are produced with relatively high frequency after fly transmission of a single strain. We have been able to demonstrate these events using mixtures of trypanosomes from a single T. b. brucei strain transfected with a gene for either red or green fluorescent protein. This strategy firstly enabled flies carrying a mixed infection of red and green fluorescent trypanosomes in the salivary glands to be identified, and secondly allowed us to detect any recombinants by yellow fluorescence directly in the fly $[7,8]$. This focused the analysis on populations likely to contain recombinants, rather than laboriously screening all populations as in previous studies. Thus, starting from 35 flies with infected salivary glands, we identified nine flies carrying a mixed infection 


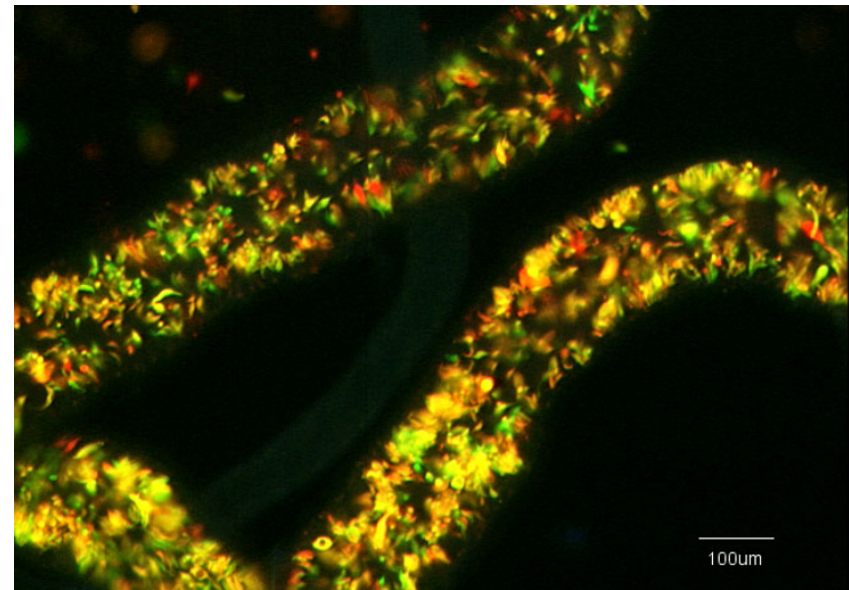

Figure 5

Infected salivary glands SG3 clone 7. Dissected salivary glands from a fly infected with the yellow fluorescent hybrid clone SG3 clone 7. Red, green and yellow fluorescent trypanosomes are present.

of red and green fluorescent trypanosomes, and were able to detect putative recombinants by yellow fluorescence in four flies, of which just two finally yielded recombinant trypanosome clones. This contrasts with the high frequency of mating observed in the outcross of these two parental strains, where hybrids were easily isolated from four flies with salivary glands containing a mixed infection of red and green fluorescent trypanosomes, and putative hybrids, as judged by yellow fluorescence, were evident in a total of 17 of $22(77 \%)$ flies with a mixed salivary gland infection [8]. Comparing the numbers of flies where yellow trypanosomes were observed in mixed salivary gland infections, at a rough estimate recombinants are half as likely to be recovered from flies carrying a single trypanosome strain (four out of nine, 44\%) compared to a mixed infection (17 of $22,77 \%$ ).

What processes are occurring during intraclonal mating? The observation of yellow fluorescent trypanosomes - in abundance in the salivary glands of one fly - indicates that fusion between individual cells occurs, although we were unable to recover any yellow recombinant trypanosomes even after drug selection. This suggests that non-viable recombinants may sometimes be formed, echoed by the observation that salivary glands from flies infected with a yellow fluorescent clone often appeared to contain a mixture of red, green and yellow fluorescent trypanosomes but green fluorescent trypanosomes were not recovered. One possibility is that a proportion of trypanosomes undergo meiosis, but the resultant intermediates then fail to produce viable diploids. Perhaps the lack of cells of a different mating type in a single strain transmission aborts the normal course of events and the intermediates simply die in situ. As mentioned earlier, there is currently no evi-

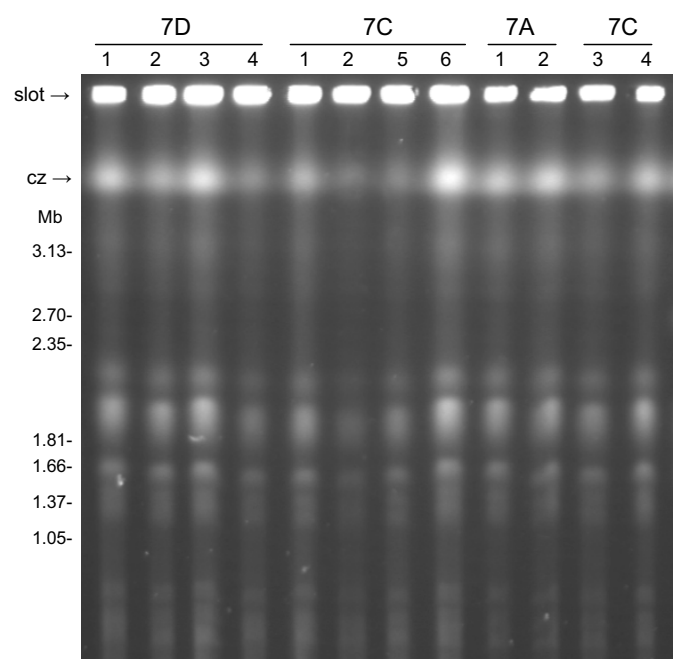

A.

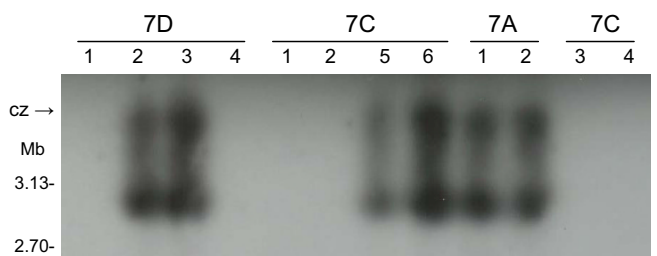

B. GFP

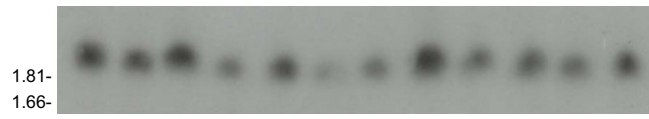

C. RFP

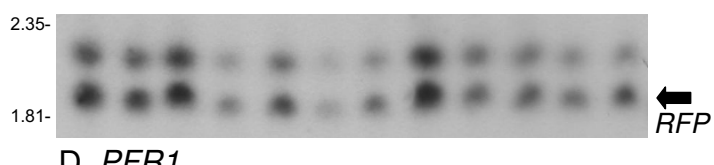

D. PFR1

\section{Figure 6}

Molecular karyotypes of clones derived after transmission of SG3 clone 7. A. Ethidium bromide stained pulsed field gel. Size marker: chromosomal DNAs from Hansenula wingei. $\mathrm{Cz}=$ compression zone, a region of the gel where several large chromosomal bands are trapped. B - D. Autoradiographs of Southern blot of same gel showing results following hybridization with the probes indicated. All blots were washed to $0.1 \times \mathrm{SSC}$ at $65^{\circ} \mathrm{C}$. In panel B the GFP gene is located on a single chromosome of approximately $3 \mathrm{Mb}$ in size, but there is also hybridization to DNA trapped in the compression zone. In panel D arrow indicates chromosome III homologue that also hybridized with the RFP gene (panel C). 
Table 5: Genotypes and DNA contents of clones from transmission of yellow fluorescent clone

\begin{tabular}{|c|c|c|c|c|c|c|c|c|c|c|}
\hline \multirow[t]{2}{*}{ Clone } & \multirow[t]{2}{*}{ Fluorescence } & \multirow[t]{2}{*}{ DNA content ${ }^{\mathrm{a}}$} & \multicolumn{8}{|c|}{ Microsatellite locus } \\
\hline & & & II-PLC & III-2 & IV-TB4/7 & VI-TB6/I & VIII-TB8/9 & IX-ТВ9/6 & X-TB $10 / 17$ & XI-53 \\
\hline SG3 clone 7 & Yellow & $2 \mathrm{Nb}^{\mathrm{b}}$ & bc & bd & bc & $a b$ & $a b$ & bc & ac & $a b$ \\
\hline \multicolumn{11}{|l|}{ Transmission I } \\
\hline 7A clone I & Yellow & 0.91 & bc & bd & bc & $a b$ & $a b$ & bc & ac & $a b$ \\
\hline 7A clone 2 & Yellow & 0.90 & bc & bd & bc & $a b$ & $a b$ & bc & ac & $a b$ \\
\hline 7C clones I-4 & Red & ND & bc & bd & bc & $a b$ & $a b$ & bc & ac & $a b$ \\
\hline $7 C$ clones 5,6 & Yellow & ND & bc & bd & bc & $a b$ & $a b$ & bc & ac & $a b$ \\
\hline 7D clones I & Red & 1.58 & bc & bd & bc & $a b$ & $a b$ & bc & ac & $a b$ \\
\hline 7D clones 2,3 & Yellow & ND & bc & bd & bc & $a b$ & $a b$ & bc & ac & $a b$ \\
\hline 7D clones 4 & Red & 0.78 & bc & bd & bc & $a b$ & $a b$ & bc & $\mathrm{ac}$ & $a b$ \\
\hline \multicolumn{11}{|l|}{ Transmission 2} \\
\hline 7AI-I clones I, 2 & Yellow & ND & bc & bd & bc & $a b$ & $a b$ & bc & ac & $a b$ \\
\hline 7AI-2 clones I, 2 & Yellow & ND & bc & bd & bc & $a b$ & $a b$ & bc & $\mathrm{ac}$ & $a b$ \\
\hline 7AI-3 clone I & Yellow & ND & bc & bd & bc & $a b$ & $a b$ & bc & ac & $a b$ \\
\hline 7AI-3 clone 2 & Red & ND & bc & bd & bc & $a b$ & $a b$ & bc & $\mathrm{ac}$ & $a b$ \\
\hline 7AI-4 clone I & Yellow & 1.42 & bb & bd & bb & $a b$ & $a b$ & bc & ac & $a b$ \\
\hline 7AI-4 clone 2 & Red & ND & bc & bd & bc & $a b$ & $a b$ & bc & cc & $a b$ \\
\hline 7A2-I clones I, 3 & Yellow & ND & bc & bd & bc & $a b$ & $a b$ & bc & ac & $a b$ \\
\hline 7A2-I clone 2 & Red & ND & bc & bd & bc & $a b$ & $a b$ & $\mathrm{bc}$ & ac & $a b$ \\
\hline 7A2-2 clones I, 2 & Yellow & ND & $b c$ & bd & bc & $a b$ & $a b$ & bc & ac & $a b$ \\
\hline
\end{tabular}

a. DNA content relative to T. b. brucei strain 427, arbitrarily assigned a DNA content of I.00. ND, not done.

b. Measured previously [8].

dence that mating types occur in T. brucei, but the contrasting numbers of recombinant/non-recombinant clones recovered from single versus two strain transmissions continues to support the idea that trypanosomes can recognise self and non-self.

After fly transmission, it was found that several clones analysed had a raised, or in one case, lowered DNA content relative to the original clone prior to fly transmission. Most of these clones also had a recombinant genotype according to microsatellite analysis, but, in three cases, no change other than in DNA content was detected, although more extensive genotyping might reveal evidence of recombination. Some of the raised DNA contents observed were consistent with triploidy or tetraploidy, but the molecular karyotype analysis revealed no evidence of extra chromosomes. This echoes the finding of hybrids with raised DNA contents in the previous outcross, where it was suggested that the putative $4 \mathrm{~N}$ hybrids might have been formed by fusion of unreduced gametes [8].

Although the overall picture is far from clear, one thing is certain: that it is no longer possible to assume that strains are fly-transmitted unaltered. This has implications for 
both laboratory and field infections, as infected glands will potentially contain a heterogeneous population of trypanosomes.

\section{Competing interests}

The authors declare that they have no competing interests.

\section{Authors' contributions}

WG, LP and MB designed the study. All authors contributed to the experimental work. WG and LP drafted the manuscript. All authors read and approved the final manuscript.

\section{Acknowledgements}

This work was supported by Wellcome Trust grant number 079375 . We thank Roger Tsien (University of California, San Diego) for supplying the mRFP construct.

\section{References}

I. Jenni L, Marti S, Schweizer J, Betschart B, Lepage RWF, Wells JM, Tait $A$, Paindavoine P, Pays E, Steinert M: Hybrid formation between African trypanosomes during cyclical transmission. Nature 1986, 322:173-175.

2. Gibson W, Stevens J: Genetic exchange in the Trypanosomatidae. Adv Parasitol 1999, 43:1-46.

3. Richner D, Brun R, Jenni L: Production of metacyclic forms by cyclical transmission of West African Trypanosoma (Trypanozoon) brucei isolates from man and animals. Acta Trop 1988, 45:309-319.

4. Gibson W, Winters K, Mizen G, Kearns J, Bailey M: Intraclonal mating in Trypanosoma brucei is associated with out-crossing. Microbiology UK 1997, I 43:909-920.

5. Tait A, Buchanan N, Hide G, Turner M: Self-fertilisation in Trypanosoma brucei. Mol Biochem Parasitol 1996, 76:3I-42.

6. Turner CMR, Sternberg J, Buchanan N, Smith E, Hide G, Tait A: Evidence that the mechanism of gene exchange in Trypanosoma brucei involves meiosis and syngamy. Parasitology 1990, I01:377-386.

7. Gibson W, Peacock L, Ferris V, Williams K, Bailey M: Analysis of a cross between green and red fluorescent trypanosomes. Biochem Soc Trans 2006, 34:557-559.

8. Gibson W, Peacock L, Ferris V, Williams K, Bailey M: The use of yellow fluorescent hybrids to indicate mating in Trypanosoma brucei. Parasites and Vectors 2008, I:4.

9. Kabayo JP: The nature of the nutritional importance of serumalbumin to Glossina morsitans. J Insect Physiol 1982, 28:917-923.

10. Galun R, Margalit J: Adenine nucleotides as feeding stimulants of tsetse fly Glossina austeni Newst. Nature 1969, 222:583-584.

II. Peacock L, Ferris V, Bailey M, Gibson W: Multiple effects of the lectin-inhibitory sugars $\mathrm{D}$-glucosamine and $\mathbf{N}$-acetyl-glucosamine on tsetse-trypanosome interactions. Parasitology 2006, 132:65I-658.

12. Macleod ET, Maudlin I, Darby AC, Welburn SC: Antioxidants promote establishment of trypanosome infections in tsetse. Parasitology 2007, 134:827-83I.

13. Gibson WC, Fase-Fowler F, Borst P: Further analysis of intraspecific variation in Trypanosoma brucei using restriction site polymorphisms in the maxi-circle of kinetoplast DNA. Mol Biochem Parasitol 1985, 15:2 I-36.

14. Gibson WC, Marshall TFdC, Godfrey DG: Numerical analysis of enzyme polymorphism: a new approach to the epidemiology and taxonomy of trypanosomes of the subgenus Trypanozoon. Adv Parasitol 1980, 18: 175-246.

15. Campbell RE, Tour O, Palmer AE, Steinbach PA, Baird GS, Zacharias $\mathrm{DA}$, Tsien RY: A monomeric red fluorescent protein. Proc Natl Acad Sci USA 2002, 99:7877-7882.

16. Cunningham I: New culture medium for maintenance of tsetse tissues and growth of trypanosomatids. J Protozool 1977, 24:325-329.
17. Van der Ploeg LH, Schwartz DC, Cantor CR, Borst P: Antigenic variation in Trypanosoma brucei analysed by electrophoretic separation of chromosome-sized DNA molecules. Cell 1984, 37:77-84.

18. Thomashow LS, Milhausen M, Rutter M, Agabian N: Tubulin genes are tandemly linked and clustered in the genome of Trypanosoma brucei. Cell 1983, 32:35-43.

19. Berriman M, Ghedin E, Hertz-Fowler C, Blandin G, Renauld H, Bartholomeu DC, Lennard NJ, Caler E, Hamlin NE, Haas B, et al:: The genome of the African trypanosome Trypanosoma brucei. Science 2005, 309:416-422.

20. MacLeod A, Tweedie A, McLellan S, Taylor S, Cooper A, Sweeney L, Turner CMR, Tait A: Allelic segregation and independent assortment in Trypanosoma brucei crosses: Proof that the genetic system is Mendelian and involves meiosis. Mol Biochem Parasitol 2005, I 43: I2-19.

21. Peacock L, Ferris V, Bailey M, Gibson W: Dynamics of infection and competition between two strains of Trypanosoma brucei brucei in the tsetse fly observed using fluorescent markers. Kinetoplastid Biology and Disease 2007, 6:4.
Publish with Bio Med Central and every scientist can read your work free of charge

"BioMed Central will be the most significant development for disseminating the results of biomedical research in our lifetime. "

Sir Paul Nurse, Cancer Research UK

Your research papers will be:

- available free of charge to the entire biomedical community

- peer reviewed and published immediately upon acceptance

- cited in PubMed and archived on PubMed Central

- yours - you keep the copyright
BioMedcentral 